\title{
Mejorando la disponibilidad de recursos educativos digitales para enseñar en escuelas rurales sin acceso a internet
}

\section{Title: Improving the availability of digital educational resources to teach in rural schools without internet access}

\author{
Manuel J. Ibarra \\ Universidad Nacional Micaela Bastidas de Apurímac, \\ Perú \\ manuelibarra@gmail.com
}

Joffre Huaman

Universidad Nacional Micaela Bastidas de Apurímac, Perú

joffrehn@hotmail.com

\author{
Pablo E. Ataucusi \\ Universidad Nacional Micaela Bastidas de Apurímac, \\ Perú \\ elicoena@hotmail.com
}

\author{
Braulio Barzola \\ Universidad Nacional Micaela Bastidas de Apurímac, \\ Perú \\ bbarzola72@hotmail.com
}

\section{Resumen}

En el Perú, la brecha digital entre los alumnos que estudian en Instituciones Educativas del área urbana y rural es bastante marcada. Este trabajo de investigación describe la fundamentación teórica de las TIC y la educación, la educación rural inclusiva y los recursos educativos abiertos; además, propone una plataforma educativa virtual, que funciona en una intranet y sin conexión a internet. Esto permite tener múltiples recursos digitales como: PeruEduca, EducaRed, EducaApurimac, Diccionario RAE, Traductor español-inglés, MateMedia y CD-Pedia, de tal manera que los docentes y alumnos puedan disponer de más recursos para el proceso de enseñanza aprendizaje. Las pruebas de usabilidad se realizaron en dos escuelas rurales de Apurímac y los resultados muestran que los docentes y alumnos están satisfechos con la herramienta propuesta.

Palabras-Clave: Brecha digital, recursos educativos abiertos, educación rural, recursos digitales sin internet.

\begin{abstract}
In Peru, the digital gap between students that study in rural and urban schools is quite marked. This paper describes the theoretical basis of ICT and education, inclusive rural education and open educational resources; In addition, it proposes a virtual educational platform, it runs into an intranet and without an internet connection. This allows to have multiple digital resources: PeruEduca, EducaRed, EducaApurimac, RAE Dictionary, SpanishEnglish translator, MateMedia and CD-Pedia, thus teachers and students can have more resources for teachinglearning process. Usability tests were conducted in two rural schools in Apurimac and the results show that teachers and students are satisfied with the proposed tool.
\end{abstract}

Keywords: Digital gap, open educational resource, rural education, off-line digital resources

Cite as: Ibarra, M., Ataucusi, P., Barzola, B., Huaman, J. (2017). Improving the availability of digital educational resources to teach in rural schools without internet access (Mejorando la disponibilidad de recursos educativos digitales para enseñar en escuelas rurales sin acceso a internet).Brazilian Journal of Computers in Education (Revista Brasileira de Informática na Educação - RBIE), 25(3), 80-94. DOI: 10.5753/RBIE.2017.25.3.80 


\section{Introducción}

En Latinoamérica, el Perú ocupa los últimos lugares en evaluación de la enseñanza y aprendizaje en las áreas de matemática y comunicación, así lo muestran las pruebas realizadas por PISA (2017), y LLECE (2014). En el Perú el Ministerio de Educación mediante la Unidad de Medición de la Calidad Educativa ha llevado a cabo la Evaluación Censal de Estudiantes ECE (2014). Los resultados obtenidos por la ECE muestran que existe una relación entre el nivel socioeconómico y los puntajes obtenidos por los estudiantes: Lima metropolitana y Callao con mayor puntaje; Ancash, Madre de Dios, Loreto y Apurímac con menor puntaje. Los resultados de la ECE también muestran que existen diferencias muy marcadas entre los logros alcanzados en las Instituciones Educativas urbanas y rurales.

Según Paredes (2010), en el ámbito rural, los departamentos con los mayores niveles de pobreza se encuentra en Apurímac (74.10\%), Puno (72.94\%) y Cusco (68.20\%). Por dominio geográfico o región natural, en la sierra el porcentaje de pobres es de $52.27 \%$, en la selva de $23.08 \%$ y en la costa de $17.18 \%$.

En el 2007, el gobierno peruano mediante el programa OLPC (por sus siglas en inglés) inició la adquisición de computadoras personales de bajo costo para los alumnos de bajos recursos económicos, ese mismo año el Ministerio de Educación a través de su Dirección General de Tecnología Educativa comenzó a entregar las computadoras portátiles XO a escuelas rurales multigrado. El programa buscó mejorar la calidad de la educación de los estudiantes de Educación Primaria en zonas rurales del país. Si bien la entrega de las laptops XO a los estudiantes en el ámbito educativo rural ofrece una serie de ventajas, principalmente referidas al acercamiento de los niños a la tecnología; sin embargo, por falta de recursos económicos en las Instituciones Educativas, no ha sido posible implementar la infraestructura adecuada para una intranet (Ver Figura 1) que permita compartir recursos en un servidor de aplicaciones, de tal manera que el docente pueda crear sus recursos educativos y compartirlos con los estudiantes.

Existen experiencias similares en el fracaso del uso de las computadoras XO. En Uruguay, el informe titulado "Profundizando en los efectos del Plan Ceibal", realizado por profesionales del Instituto de Economía de la Udelar y financiado por el propio plan y por la Administración Nacional de Educación Pública (ANEP), analizó detalladamente el plan CEIBAL y afirma que la distribución de computadoras portátiles no ha generado una mejora en los resultados académicos de los estudiantes (De Melo, Machado, Miranda \& Viera, 2013); también es necesario implementar un modelo de alfabetización para que el plan CEIBAL tenga éxito (Ceretta \& Picco, 2013). Similarmente el proyecto Canaima Educativo en la República Bolivariana de Venezuela, brindó computadoras a alumnos de primer grado; sin embargo, se conoce que aún faltan verdaderos mecanismos de alfabetización en informática, no sólo para provocar la apropiación tecnológica sino para garantizar el uso apropiado de la tecnología disponible (Zabala, Zabala \& Reyes, 2013).

La educación en el ámbito rural supone un reto para la creatividad y la innovación docente, el cual viene impulsado por el propio entorno. La educación rural tiene características particulares como: lejanía de la Institución Educativa respecto a la ciudad, zonas inaccesibles por vehículos motorizados, escasos recursos económicos y educativos, entre otros. 


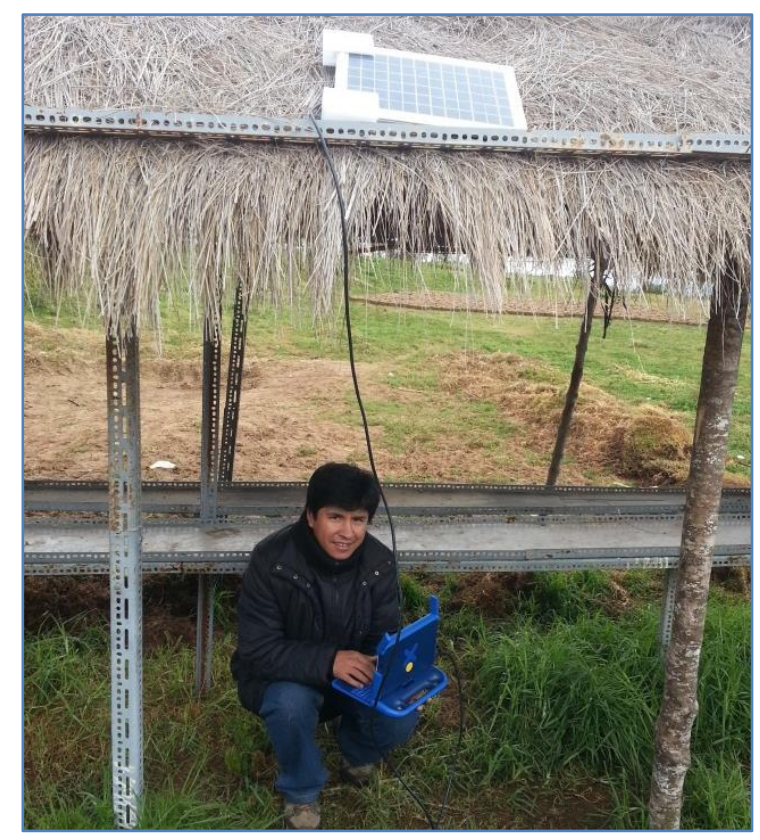

Figura 1. Laptop "XO" funcionando con panel solar en un colegio rural en Apurímac

El problema es que la brecha digital existente entre la zona urbana (zona favorecida) y la zona rural (zona desfavorecida) es cada vez mayor, en vista que en la zona rural no tienen las mismas condiciones de acceso a las Tecnología de Información y Comunicación (TIC), es así que existen escuelas rurales que no tienen una conexión a internet, por tanto, no es posible acceder a recursos digitales en línea. Según el Instituto Nacional de Estadística e Informática del Perú-INEI la brecha digital entre la zona urbana y rural se ha mantenido constante en los últimos 8 años, ver Tabla 1.

Tabla 1. Evolución del acceso a las TIC según INEI

\begin{tabular}{|c|r|r|r|r|r|r|r|r|}
\hline Área & $\mathbf{2 0 0 7}$ & $\mathbf{2 0 0 8}$ & $\mathbf{2 0 0 9}$ & $\mathbf{2 0 1 0}$ & $\mathbf{2 0 1 1}$ & $\mathbf{2 0 1 2}$ & $\mathbf{2 0 1 3}$ & $\mathbf{2 0 1 4}$ \\
\hline Urbana & 40.1 & 40.2 & 42.8 & 43.5 & 42.8 & 47.3 & 48.3 & 49.2 \\
\hline Rural & 7.4 & 8.5 & 9.2 & 9.9 & 9.1 & 10.4 & 10.9 & 11.5 \\
\hline
\end{tabular}

Si bien las TIC ofrecen herramientas pedagógicas para el docente, éstas requieren estar acompañadas de un cambio sustancial en la dirección del proceso de enseñanza-aprendizaje (didáctica), esto ha sido ampliamente discutido por investigadores de la especialidad como: Caviedes (2014); (Rivero, Padrón \& Izaguirre, 2012); (Santos Carrazana \& Carrazana Quintero, 2015); Martinez (2015); (Escorcia-Oyola \& De Triviño, 2015); (Ibarra, Soto, Ataucusi \& Ataucusi, 2016).

Este artículo presenta una estrategia para resolver el problema anteriormente planteado, de tal manera que se pueda implementar una plataforma educativa que contenga recursos educativos digitales para que el maestro y sus estudiantes los puedan utilizar; también es posible que los maestros puedan crear sus propios materiales educativos y subirlos al servidor para que posteriormente puedan compartir dicha información dentro de su escuela. 


\section{Trabajos relacionados}

Los investigadores (Kulkarni, Ballal \& Gawade, 2012), realizaron un trabajo denominado "Technology Transfer to rural population through secondary schools: The Vigyan Ashram experience". El estudio se realizó en la India y muestran que existe una diferencia notable entre la educación en la zona urbana y la zona rural, saben que el uso de nuevas tecnologías para la generación de medios de vida puede ayudar a aumentar la productividad y la eficiencia; sin embargo, hay una serie de dificultades en la transferencia de nuevas tecnologías a los estudiantes en la zona rural. Los autores desarrollaron una nueva forma de transferir tecnología en la zona rural y lo denominaron el programa Transferencia de Tecnología Básica (IBT por sus siglas en inglés). Los resultados muestran que los alumnos desarrollaron pequeños cursos que les permitió resolver problemas de la vida cotidiana.

Por otro lado, Cheng et al (2012), realizaron una investigación que lleva por título "Opportunities for social innovation at the intersection of ICT education and rural supply chains". El estudio se realizó en la India y trata de la creación de un framework para combinar el sistema educativo y la cadena de producción rural por medio de las Nuevas Tecnologías de Información y Comunicación (NTIC). Los resultados muestran que el framework es funcional y que las TIC fueron un medio importante para la creación de cursos mediante la plataforma Moodle.

Los investigadores (Chakraborty, Bhattacharya, Bhowmick, Basu \& Sarkar, 2007), llevaron a cabo la investigación titulada "Shikshak: An Intelligent Tutoring System Authoring Tool for Rural Education”. El estudio consistió en implementar un sistema tutor para la educación en colegios rurales que tienen bajo nivel de alfabetización en India. Los contenidos educativos fueron creados para el nivel primario de primero a cuarto grado, por ejemplo se hicieron pruebas con los cursos de física, ciencia, geografía e historia. Los resultados del sistema implementado muestran buenos efectos en los alumnos; sin embargo, falta resolver el problema de apagones que en algunas escuelas rurales surgen cortes temporales en el funcionamiento del fluido eléctrico.

De otro lado, (Ibarra, Ataucusi \& Ataucusi, 2015), realizaron una investigación denominada "EducaApurimac una plataforma educativa con múltiples recursos digitales para enseñar en escuelas rurales sin acceso a internet". Esta investigación realizó una primera propuesta para crear una plataforma educativa virtual con múltiples recursos educativos digitales como: PeruEduca, EducaRed, EducaApurimac, Diccionario RAE, Traductor español-inglés y MateMedia. Sin embargo, en esta investigación faltó precisar la fundamentación teórica de los conceptos importantes como por ejemplo: aprendizaje virtual, la creación de recursos educativos digitales abiertos y la educación rural inclusiva.

Después de revisar los trabajos previos realizados, se puede apreciar que existen iniciativas de querer reducir la brecha digital existente entre la zona urbana y zona rural; para esto se usan estrategias que puedan propiciar el uso de las TIC en el área rural en el ámbito económico y social. El trabajo de investigación más próximo al que planteamos es el de Ibarra et al. Esta propuesta plantea fundamentar teóricamente la estrategia para implementar una plataforma educativa en escuelas del ámbito rural del Perú, en las que los estudiantes y profesores no tienen acceso a Internet y viven aislados de la tecnología, a pesar que tienen el hardware disponible, pero no cuentan con el software que les permita interactuar para compartir materiales educativos con los estudiantes. 


\section{Fundamentación Teórica}

\subsection{Las TIC y la educación}

La capacidad de incorporar las TIC a la educación, da la posibilidad de acercar conocimientos a más lugares y personas y supone también una innovación en la educación. Al existir más posibilidades, el aprendizaje se ve modificado en comparación con una enseñanza más tradicional. Las prácticas educativas sufren una transformación, porque el uso de las TIC ofrecen diferentes opciones que el docente pueda complementar su enseñanza con el uso de las computadoras y el alumno pueda hacer su aprendizaje de manera divertida y dinámica.

La inclusión de las TIC en el aula implica un cambio en el rol del profesor y del estudiante, donde el primero adopta el papel de facilitador del aprendizaje y el segundo el papel de sujeto activo del aprendizaje. Se espera además que la integración de las TIC favorezca un cambio en las metodologías, actividades y evaluaciones del proceso de enseñanza-aprendizaje, desde el trabajo individual y basado en la memorización, hacia un trabajo colaborativo y basado en la elaboración personal del conocimiento (Claro, Espejo, Jara \& Trucco, 2011).

En términos generales, la propuesta de uso de las TIC en la educación pública se basa, entre otras, en las siguientes premisas:

-Las tecnologías digitales (en particular las computadoras) no representan por sí mismas una transformación pedagógica. Para que esto último ocurra debe existir un sustento pedagógico orientado a toda la comunidad educativa; esto es, una propuesta de uso de las TIC dentro del proceso educativo.

-La idea general del uso de las TIC es trascender en la alfabetización digital de la población y el aprendizaje sobre el uso de las tecnologías digitales y por otro lado convertirlas en recursos al servicio de una experiencia pedagógica enriquecida con la participación de estudiantes, docentes y comunidades educativas.

-Dentro de un proceso de enseñanza-aprendizaje de calidad, las TIC pueden apoyar significativamente en el desarrollo de las denominadas competencias del siglo XXI.

\subsection{Educación rural inclusiva}

En algunos países de América Latina, la brecha digital existente entre la educación en la zona urbana y la zona rural ha sido bastante marcada. Se ha podido notar que, desde hace un par de décadas, las TIC han permeado los sistemas escolares del mundo entero con la promesa de mejorarlos (Sunkel \& Trucco, 2012); Claro (2011).

Las escuelas rurales han hecho esfuerzos para no permanecer al margen de esta tendencia global. A fines de la década de los ochenta y principios de los noventa, se comenzaron a gestar las primeras políticas y programas de TIC orientados a mejorar la calidad educativa de las Instituciones Educativas, así lo manifiesta (Sunkel \& Trucco, 2012).

Según Tedesco (1999), la rigidez, la distancia y el aislamiento institucional de la escuela, la persistencia de prácticas pedagógicas tradicionales (Carneiro, Toscano \& Diaz, 2009), la falta de una adecuada integración de las TIC en el currículo (Avendaño-Castro \& Parada-Trujillo, 2013) y la escasa formación de directivos escolares en entornos TIC, son algunas de las principales condiciones y factores que afectan las buenas prácticas pedagógicas y el buen uso de las TIC en las escuelas rurales.

La educación inclusiva responde a un enfoque filosófico, social, económico, cultural, político y pedagógico que persigue la aceptación y valoración de las diferencias en la escuela para cada uno de los alumnos, Castillo et al., (2014). Así también, en la educación inclusiva los 
alumnos se benefician de una enseñanza adaptada a sus necesidades Castillo et al., (2014); (Marchesi, Blanco, Hernández \& Educativas, 2014).

Dentro de este marco se plantea la necesidad de repensar la práctica docente, proponiendo nuevos desafíos que permitan generar, entre otros aspectos, estrategias pedagógicas alternativas para la construcción de: respuestas a las necesidades educativas para las personas con barreras para el aprendizaje, su participación en distintos contextos, la promoción de las alfabetizaciones múltiples y el aprendizaje constructivo.

\subsection{Aprendizaje virtual}

Actualmente, existe una corriente que habla de entornos más abiertos de formación que ofrecen más posibilidades a más usuarios y usuarias. Otros términos que se están introduciendo cada vez más en estos ámbitos de actuación, son las denominadas Comunidades Virtuales de Aprendizaje (CVA) o los entornos de aprendizaje en línea e-learning.

Dependiendo del grado de presencialidad o distancia en la interacción entre profesor y alumno (Area, San Nicolás \& Fariña, 2010). se diferencian tres modelos de enseñanza:

- Modelo de enseñanza presencial: en esta modalidad de enseñanza las TIC es en forma presencial y se integran como recursos de apoyo a los procesos de enseñanza/aprendizaje.

- Modelo de enseñanza a distancia: los entornos virtuales de aprendizaje (EVA) o entornos virtuales de formación median los procesos de enseñanza a distancia, aportando información y recursos pedagógicos para mejorar la calidad de los aprendizajes y permitir un "acercamiento" entre profesores y alumnos.

- Modelo de enseñanza semipresencial: Es una combinación del modelo de enseñanza presencial y a distancias (blended learning).

El carácter virtual reside en el hecho de que son comunidades de aprendizaje que utilizan las TIC en una doble vertiente: como instrumentos para facilitar el intercambio y la comunicación entre sus miembros, y como instrumentos para promover el aprendizaje (Bustos \& Coll 2010); (Hinojo \& Fernández, 2012).

\subsection{Recursos Educativos Abiertos}

El término "recursos educativos abiertos"-en inglés, open educational resources (OER)- fue acuñado en 2002 por la UNESCO (Santos-Hermosa, Ferran-Ferrer \& Abadal, 2012). Según la OECD (2007), los OER son los materiales y recursos educativos que pueden ser reutilizados para la docencia y el aprendizaje de forma gratuita. Las definiciones de los OER varían en función del aspecto que se quiere enfatizar, ya sea la reutilización o el ámbito de aplicación. Los OER son un denominador genérico que incluye cursos y programas curriculares, módulos didácticos, guías de estudiante, libros digitales, artículos de investigación, vídeos, podcasts, herramientas de evaluación, materiales interactivos (como simulaciones), bases de datos, software, aplicaciones (incluyendo aplicaciones móviles) y cualquier otro material educativo diseñado para uso en la enseñanza y el aprendizaje, Santos-Hermosa et al. (2012).

Según Santos-Hermosa et al. (2012), las principales características que deben tener los OER son:

- La accesibilidad, entendida como la disponibilidad del recurso a ser localizado y utilizado en cualquier lugar o momento.

- La reusabilidad, propiedad a ser modificado y utilizado en diferentes contextos de aprendizaje. 
- La interoperabilidad, o facilidad de ser adaptado e interconectado entre diferentes dispositivos o herramientas.

- La sostenibilidad, funcionamiento correcto a pesar de los cambios de versiones, de software.

- Los metadatos, o descripciones que posibilitan su indexación, almacenamiento, búsqueda y recuperación.

La UNESCO (2012), en junio del 2012 en París se realizó el Congreso Mundial de Recursos Educativos Abiertos, que emitió la Declaración de París sobre este tema, con un conjunto de recomendaciones para los Estados y sintetizan en:

- Fomentar el conocimiento y el uso de los recursos educativos abiertos.

- Crear entornos propicios para el uso de las TIC; apoyar el aumento de capacidades para el desarrollo sostenible de materiales de aprendizaje de calidad.

- Promover la elaboración y adaptación de recursos educativos abiertos en una variedad de idiomas y de contextos culturales.

- Facilitar la búsqueda, la recuperación y el intercambio de recursos educativos abiertos.

- Alentar la investigación sobre los recursos educativos abiertos, entre otros.

\section{Diseño e implementación de la plataforma educativa}

\subsection{Arquitectura lógica}

Los usuarios (alumnos, docentes y directores) pueden utilizar la aplicación de tres formas: a) desde el laboratorio de cómputo, en la cual se tiene computadoras conectadas con una topología estrella dentro de una red LAN; b) desde el campus de la escuela mediante una conexión WiFi, para lo cual se tiene una Antena que permite irradiar la señal hasta 1 kilómetro; c) desde su casa siempre y cuando llegue la señal.

Los usuarios que utilizan una computadora personal, utilizan un navegador web e invocan a una página web mediante el protocolo HTTP, esta página web reside en el servidor web, que a su vez peticiona los recursos necesarios para dar respuesta a la información solicitada por el cliente. Los usuarios que utilizan un dispositivo móvil, descargan un archivo del servidor e luego lo instalan en su celular o tablet. El instalador está disponible para dispositivos móviles con sistema operativo Android, iOS o Windows Mobile, ver Figura 2.

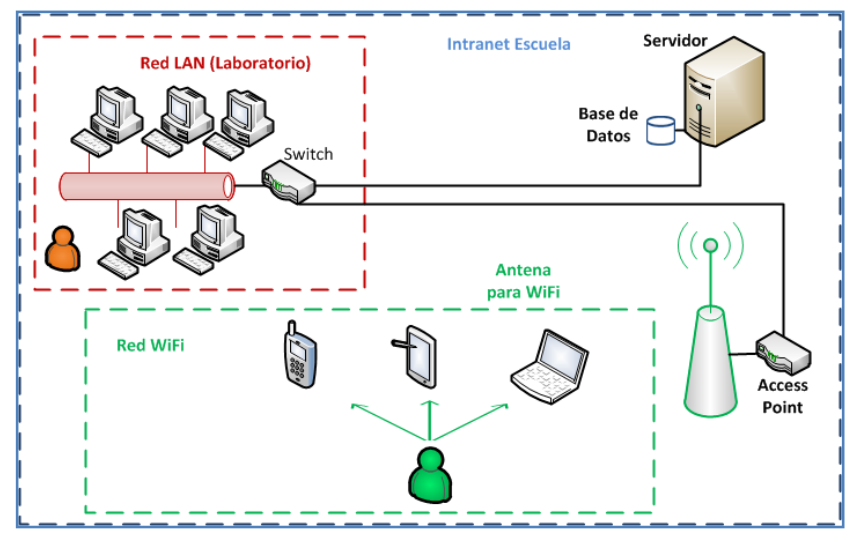

Figura 2. Arquitectura lógica de la propuesta

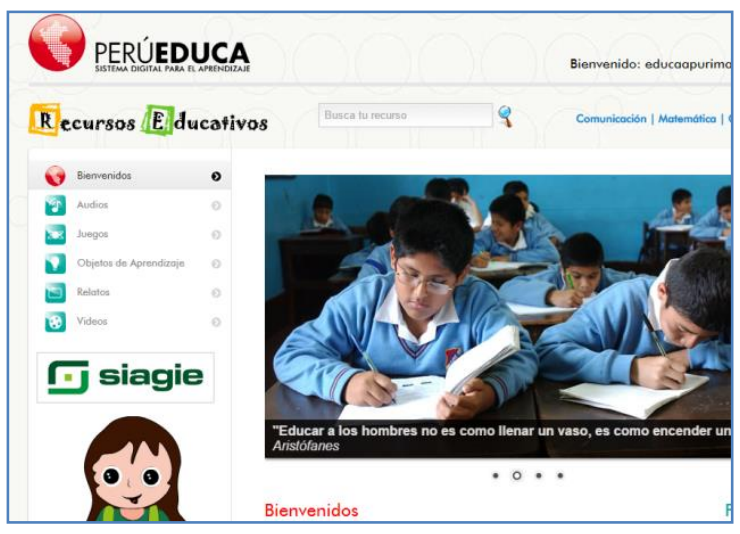

Figura 3. Módulo PerúEduca 


\subsection{Módulos que tiene la plataforma educativa que funcionan sin conexión a internet}

La plataforma educativa de aprendizaje propuesta en este trabajo de investigación tiene los siguientes módulos educativos: PeruEduca, EducaRed y EducaApurimac a continuación describimos cada uno de ellos.

\section{a) Módulo de Perú Educa}

Este módulo es una recopilación de contenidos educativos que el Ministerio de Educación del Perú ha proporcionado para que los colegios a nivel nacional puedan utilizarlo. Este módulo ha sido adaptado al servidor de la escuela para que pueda servir como recursos educativos para las escuelas rurales que no tienen conexión a internet (off-line). El módulo tiene cerca de 300 recursos educativos digitales para el nivel primario y cerca 450 recursos para el nivel secundario. Estos recursos generalmente están orientados para enseñar al alumno y docente un tema, por ejemplo: "alimentación sana". Los recursos son de uso libre (donado por Intel) están en formato de: video, animación, imagen, archivos pdf, entre otros. Ver Figura 3.

Adicionalmente, este módulo tiene recursos digitales para docentes, padres de familia, recursos multimedia, etc. También tiene la opción para que el docente pueda subir un recurso que él mismo lo elaboró a través de un repositorio digital. Así mismo, el módulo tiene contenidos educativos auspiciados por telefónica y que son de libre uso, por ejemplo EducaRed, ver Figura 4.

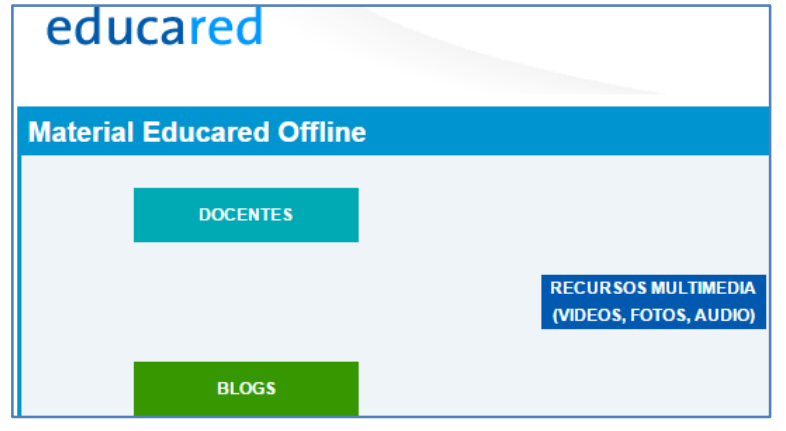

Figura 4. Módulo EducaRed

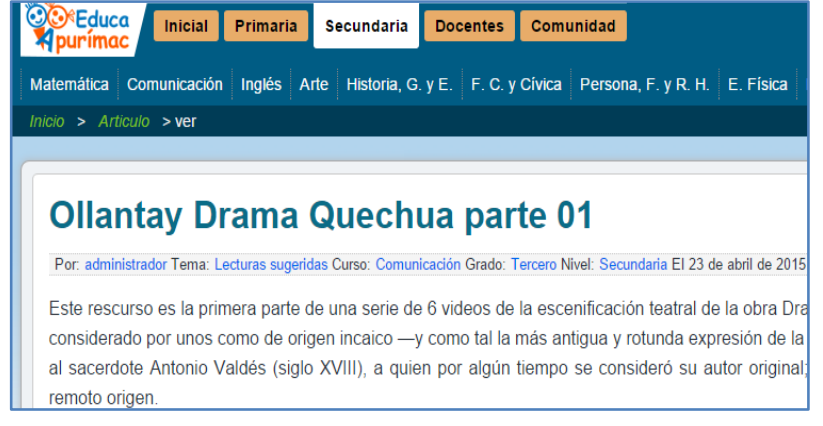

Figura 5. Módulo mis contenidos

\section{b) Módulo EducaApurimac}

Este es el módulo más importante en el desarrollo de la investigación y consta de:

b1) Módulo mis contenidos. Tiene recursos didácticos educativos para nivel "primaria", "secundaria", "docentes" y "comunidad". Además, tiene una opción para que los docentes y estudiantes puedan publicar sus contenidos, ver Figura 5.

b2) Módulo diccionario RAE off-line. Este módulo permite tener una distribución de un diccionario de la Real Academia Española (RAE) en versión off-line. Para esto la RAE dio el permiso para poder usar las palabras y su definiciones, mas no las imágenes. Entonces para poner en funcionamiento el diccionario, se descargó de la página web de la RAE, la mayor cantidad posible de palabras y luego se exportó la información obtenida a la base de datos en Mysql. En el diccionario existen alrededor de noventa mil palabras y su respectivo significado, la Figura 6 muestra el resultado de una búsqueda. 


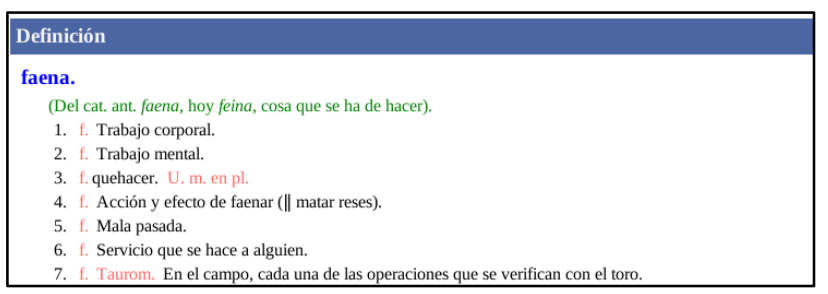

Figura 6. Módulo diccionario RAE off-line

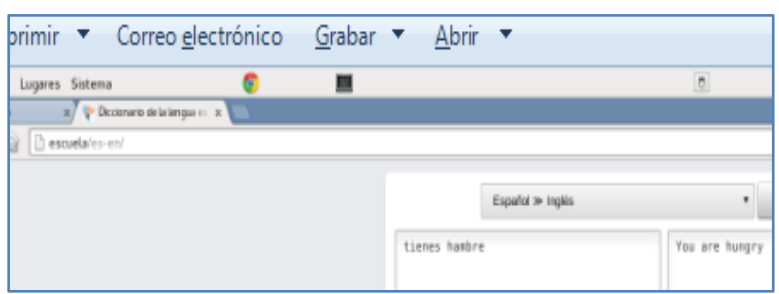

Figura 7. Traductor Español-Inglés

\section{b3) Módulo traductor de español a inglés.}

El traductor español-inglés está basado en la plataforma Apertium que es un sistema de traducción automática que ha sido desarrollado con el financiamiento conjunto del gobierno español y la Generalidad de Cataluña en la Universidad de Alicante. Es un aplicativo que pertenece a la gama de "software libre" liberado bajo los términos de la licencia GNU GPL. Este motor funciona en modo consola, pero se tuvo que desarrollar una interfaz gráfica para que pueda ser más intuitiva y sobre todo funcional, ver Figura 7.

\section{b4) Módulo Matemedia}

Son recursos digitales elaborados por el grupo de investigación, para que el alumno pueda reforzar el aprendizaje de los números naturales mediante juegos educativos tanto para el nivel primaria como para el nivel secundaria, estos recursos han sido agregados a un repositorio para que puedan ser visualizados por los alumnos de la Escuela, ver Figura 8.

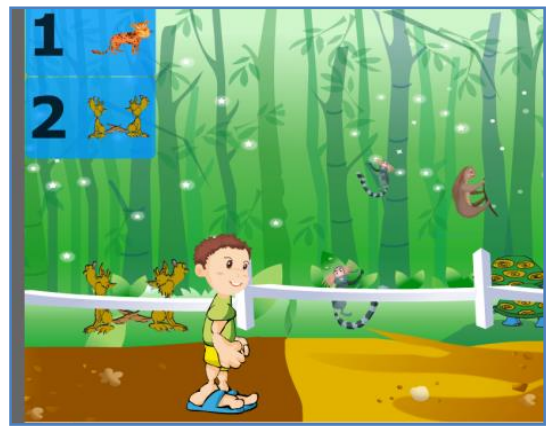

Figura 8. Módulo Matemedia

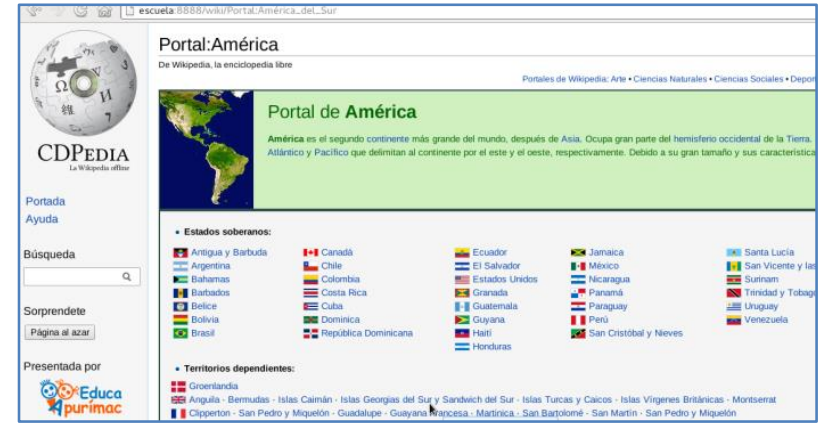

Figura 9. CD-Pedia en funcionamiento

\section{b5) Módulo CD-Pedia}

CD-Pedia es un proyecto desarrollado por la comunidad de usuarios de Python de Argentina (PyAr) para disponer de la información de "Wikipedia" en idioma español sin necesidad de conexión a Internet. A fines de junio de 2011 se lanzó la versión 0.7 de CDPedia en DVD y por medio de un acuerdo con Wikimedia Argentina, Python Argentina (PyAr) y Educ.ar se distribuyó en las escuelas de Argentina, bajo licencias que permiten su copia libre. También se puede descargar libremente de la red y grabar a CD o DVD para repartirlos sin restricciones. CD-Pedia funciona en cualquier computadora, ya sea que tenga GNU/Linux, MacOS o Windows como sistema operativo.

Este módulo fue descargado de la página de la comunidad de PyAr, luego de haber instalado y configurado en el servidor web, el cual ya está disponible para su funcionamiento en la plataforma educativa propuesta. La Figura 9 muestra CD-Pedia en funcionamiento. 


\subsection{Características del servidor}

El servidor de la Escuela tiene las siguientes características a nivel de software: sistema operativo Fedora 14, Nucleo Linux 2.6.35.14, lenguaje de programación PHP 5.x, gestor de base de datos Mysql 5.x, validación en el cliente mediante JavaScript, HTML el lenguaje de mar-cado y CSS para los estilos de la página; a nivel hardware el servidor tiene un procesador AMD Phenom de doble núcleo (similar a core dos duo de Intel), memoria RAM de 3 GB, Disco duro de $320 \mathrm{~GB}, 2$ tarjetas de red y adicionalmente periféricos simples como monitor, teclado y mouse.

\section{Proceso de evaluación de la estrategia propuesta}

\subsection{Participantes de evaluación}

Para dar validez a esta propuesta, se aplicó un cuestionario de usabilidad a alumnos y docentes en la Institución Educativa Primaria del área rural "54461-Virgen del Carmen", con código modular "0200758", ubicada en la localidad de Saywite, en el distrito de Curahuasi de la Provincia de Abancay y Departamento de Apurímac, Perú. Los participantes fueron alumnos y docentes de cuarto, quinto y sexto grado del nivel primario. La aplicación del instrumento se realizó en el mes de abril del 2015. Participaron en total 38 personas, de los cuales 36 fueron estudiantes ( 8 alumnos de cuarto, 14 alumnos de quinto grado y 14 alumnos de quinto grado) y 2 profesores (1 profesor de quinto grado y otro profesor de sexto grado). Los profesores contaban con varios años de experiencia en el aula. Se priorizó pruebas con alumnos de los últimos grados del nivel primario, porque eran los que más acceso tenían a las computadoras.

\subsection{Proceso de experimentación}

Los docentes y alumnos utilizaron la herramienta aproximadamente por una hora, en la cual sin mucha explicación verbal, sino más bien cada uno de manera intuitiva empezó a explorar cada uno de los recursos educativos digitales, ver Figura 10 y Figura 11.

Dentro de la Institución Educativa, los alumnos tuvieron acceso a la herramienta desarrollada de varias formas: a) mediante computadoras desktop instaladas en el laboratorio de cómputo; b) mediante las computadoras XO que donó el gobierno en años pasados, c) mediante computadoras personales class-mate donadas por el gobierno el año pasado; y d) mediante dispositivos móviles celulares o tablets con sistema operativo Android. Fuera de la Institución Educativa, los alumnos también tienen la opción de utilizar la herramienta mediante una conexión WiFi de hasta $1 \mathrm{Km}$ de alcance. Finalmente, los usuarios completaron un cuestionario que buscaba evaluar la usabilidad y utilidad de la herramienta propuesta.

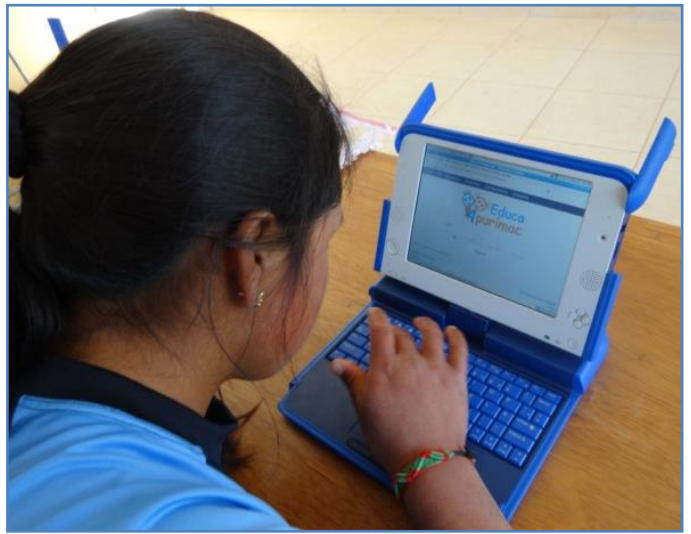

Figura 10. Accediendo desde una XO

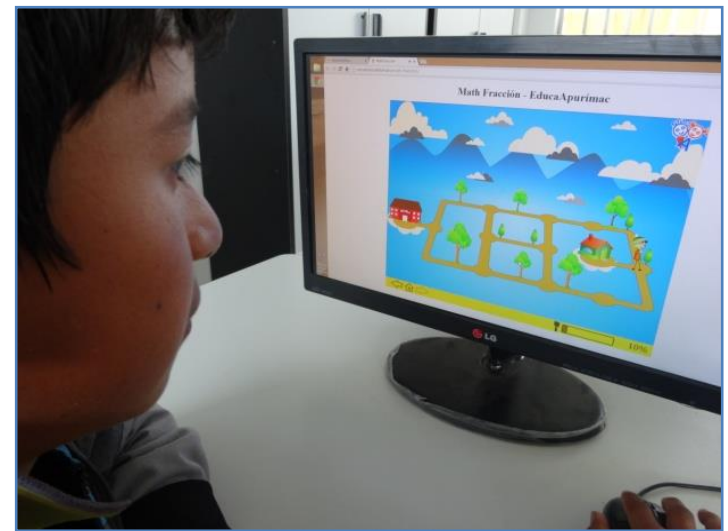

Figura 11. Accediendo desde una Desktop 


\subsection{Criterios para la usabilidad}

Para medir la usabilidad se ha tomado como referencia los criterios utilizados por (Marzal, Colmenero-Ruiz, \& Morato Lara, 2003). Dentro de los criterios más importantes están: amigabilidad, flexibilidad, diseño multimedia e interactividad. Estas variables están consideradas dentro de las preguntas de usabilidad del cuestionario utilizado para validar la propuesta. Las preguntas del cuestionario para medir la usabilidad fueron validadas de acuerdo a una prueba piloto que previamente se realizó, para esto se consideró el contexto en la cual viven los alumnos. Después de aplicar la prueba piloto con 10 usuarios, se realizó algunas correcciones en cuanto a redacción y contextualización; finalmente se aplicó de acuerdo a las preguntas mostradas en la Tabla 2.

\subsection{Resultados obtenidos}

La Tabla 2 muestra los resultados de cuestionario aplicado a los docentes y alumnos que utilizaron la herramienta.

Como se mencionó antes, en dicha sesión de aprendizaje participaron 38 personas de la Institución Educativa Virgen del Carmen. Los participantes respondieron utilizando una escala de Likert 1 a 5, donde 1 representa "Totalmente en desacuerdo", 2 representa "En desacuerdo", 3 representa "Ni de acuerdo ni en desacuerdo", 4 representa "De acuerdo" y 5 representa "Totalmente de acuerdo".

La primera columna de la tabla muestra la "Pregunta" que indica cada una de las preguntas realizadas en el cuestionario de usabilidad, luego se muestra la variable "Nro. pers", que representa el número de personas que han participado y dado una respuesta a una cierta pregunta. Luego se muestra el valor máximo (max.) y mínimo (min) asignado a ese ítem por parte de los participantes. La columna "Media" indica el promedio obtenido por la puntuación de cada participante. Finalmente, la última columna muestra la desviación estándar de los valores obtenidos.

Tabla 2. Preguntas de usabilidad para validar la propuesta

\begin{tabular}{|l|r|r|r|r|c|}
\hline \multicolumn{1}{|c|}{ Pregunta } & $\begin{array}{c}\text { Nro. } \\
\text { Pers. }\end{array}$ & $\begin{array}{c}\text { Valor } \\
\text { Max. }\end{array}$ & $\begin{array}{c}\text { Valor } \\
\text { Min. }\end{array}$ & $\begin{array}{c}\text { Me- } \\
\text { dia }\end{array}$ & $\begin{array}{c}\text { Desv. } \\
\text { Est. }\end{array}$ \\
\hline 1)Me gusta los recursos educativos de la plataforma virtual. & 38 & 5 & 3 & 4.29 & 0.60 \\
\hline 2)Es fácil de usar la plataforma educativa. & 38 & 5 & 4 & 4.57 & 0.42 \\
\hline 3)Recomendaría el uso de la plataforma educativo a otros amigos. & 38 & 5 & 3 & 4.12 & 0.55 \\
\hline 4)Los botones de desplazamiento son fáciles de identificar. & 38 & 4 & 3 & 3.17 & 0.95 \\
\hline 5)La distribución del menú está bien diseñado. & 38 & 4 & 4 & 3.94 & 0.37 \\
\hline 6)La plataforma educativa tiene bastante material educativo. & 38 & 4 & 3 & 4.01 & 0.38 \\
\hline 7)Puedo conectarme a la plataforma educativa desde cualquier lugar. & 38 & 4 & 3 & 3.77 & 0.80 \\
\hline
\end{tabular}

A continuación se analizan los resultados presentados en la Tabla 2:

Respecto a la satisfacción con la plataforma educativa, en los ítems 1) 2) 3) y 6) se obtuvo un promedio de 4.29, 4.57, 4.12 y 4.01 respectivamente. Esto nos muestra que los docentes y alumnos coinciden en mostrar su satisfacción con la plataforma educativa, porque les permite tener disponible recursos didácticos que antes no lo tenían.

Respecto al diseño del sitio web, en los ítems 4) y 5) se obtuvo un promedio de 3.17 y 3.94 respectivamente. Los docentes y alumnos califican positivamente el hecho que la aplicación mostrada tiene un buen diseño y pueden navegar sobre ella sin dificultades. 
Finalmente, respecto a la posibilidad de conexión al servidor, en el ítem 7) se obtuvo un promedio de 3.77, lo cual indica que los usuarios manifiestan que pueden conectarse en cualquier lugar dentro de la escuela, esto debido a la conexión inalámbrica.

\section{Conclusiones y trabajo futuro}

Este artículo describe conceptos teóricos referidos a las TIC y la educación, educación rural inclusiva y recursos educativos abiertos; asimismo, plantea un nuevo enfoque para implementar una plataforma educativa con recursos educativos de aprendizaje, como PeruEduca, EducaRed, EducaApurimac, Diccionario RAE, Traductor español-inglés, diversos recursos didácticos de MateMedia y CD-Pedia. La particularidad es que todos estos recursos funcionan de manera offline en la intranet de una escuela rural en Apurímac, es decir, sin necesidad de conexión a internet.

Este proyecto es un aporte para reducir la brecha digital existente entre escuelas del ámbito urbano y rural. Después de realizar las pruebas de usabilidad, una de las conclusiones obtenidas es que: los docentes y alumnos cuentan con un servidor con recursos educativos para que los docentes y alumnos puedan hacer uso y así complementar el aprendizaje en el área de las matemáticas.

Por otro lado, los alumnos y docentes se sienten satisfechos con el diseño de la interfaz web y consideran que la plataforma educativa proporciona suficiente material educativo para poder explorar y crear conocimiento propio.

Para los próximos estudios, como trabajo futuro, se pretende crear un servidor en una placa diminuta, portable y de bajo consumo de energía eléctrica, o también un instalador CD-Live o USB-Live que permita incorporar todos los recursos educativos en un solo dispositivo, de tal manera que, se pueda distribuir a otras escuelas para que hagan uso del material elaborado. Esto permitirá que cada vez más alumnos y docentes sean beneficiadas con este trabajo. Por otro lado ahora se está trabajando en un proyecto para agregar mapas que funcionen de manera off-line para la enseñanza de Geografía; además, se pretende agregar más palabras al diccionario y al traductor español inglés, asimismo, seguir trabajando en aumentar los recursos educativos para Matemedia.

\section{Referencias}

Area, M., San Nicolás, M. \& Fariña, B. (2010). Buenas prácticas de aulas virtuales en la docencia universitaria semipresencial. Teoría de la Educación. Educación y Cultura en la Sociedad de la Información, 11(3), 7-31. Retrieved from http://oai.redalyc.org/articulo.oa?id=201014897002 [GS Search]

Avendaño-Castro, W.R. \& Parada-Trujillo, A. E. (2013). El currículo en la Sociedad del Conocimiento. Educación y Educadores, 16(1), 159-174. doi: 10.5294/edu.2013.16.1.10

Bustos, A. \& Coll, C. (2010). Los entornos virtuales como espacios de enseñanza y aprendizaje. Una perspectiva psicoeducativa para su caracterización y análisis. Revista Mexicana de Investigación Educativa, 15(.44), páginas 163-184. Retrieved from http://www.scielo.org.mx/scielo.php?script=sci arttext\&pid=S1405$\underline{66662010000100009 \& \operatorname{lng}=\mathrm{es} \& \mathrm{t} \operatorname{lng}=\mathrm{es}}$

Carneiro, R., Toscano, T. C., \& Diaz, T. (2009). Los desafíos del cambio educativo. Colección Metas 2021, Fundación Santillana, España. 
Castillo, E. S., Fus, C. D., Sosa Zitto, R., Rapallini, U. M. A., Velazquez, E., Blanc, R. L., \& Lepratte, L. O. (2014). Modelos interactivos de aprendizaje basado en tecnología para la inclusión social de bajo costo y aplicaciones multi-usuarios. In XVI Workshop de Investigadores en Ciencias de la Computación. [GS Search]

Caviedes, J.F.R. (2014). La integración educativa de las nuevas Tecnologías de la Información y la Comunicación. Revista latinoamericana de estudios educativos, 44(1). Retrieved from http://4www.redalyc.org/articulo.oa?id=27030478004 [GS Search]

Ceretta, M. G. \& Picco, P. (2013). La necesidad de definir un modelo de alfabetización en información para el Plan Ceibal. TransInformacao, 25(2). Retrieved from http://periodicos.puc-campinas.edu.br/seer/index.php/transinfo/article/view/1952 [GS $\underline{\text { Search }]}$

Chakraborty, S., Bhattacharya, T., Bhowmick, S., Basu, A., \& Sarkar, S. (2007). Shikshak: An intelligent tutoring system authoring tool for rural education. Information and Communication Technologies and Development, ICTD, 1-10. doi: 10.1109/ICTD.2007.4937410

Cheng, A., Sinha, A., Shen, J., Mouakkad, S., Joseph, L., \& Mehta, K. (2012). Opportunities for social innovation at the intersection of ICT education and rural supply chains. In Global Humanitarian Technology Conference (GHTC) IEEE, 328-335. doi: $\underline{10.1109 / \mathrm{GHTC} .2012 .81}$

Claro, M. (2011). El papel de las tecnologías de la información y las comunicaciones en la educación inclusiva. Documentos de Proyectos, 434. Retrieved from http://repositorio.cepal.org/handle/11362/3937 [GS Search]

Claro, M., Espejo, A., Jara, I. \& Trucco, D. (2011). Aporte del sistema educativo a la reducción de las brechas digitales. Una mirada desde las mediciones PISA, Documento de proyecto, (LC/W456), Santiago de Chile, Comisión Económica para América Latina y el Caribe (CEPAL). Retrieved from http://repositorio.cepal.org/handle/11362/3958 [GS Search]

De Melo, G., Machado, A., Miranda, A. \& Viera, M. (2013). Profundizando en los efectos del Plan Ceibal. Instituto de Economía (UDELAR) y Centro de Investigación y Docencia Económicas (CIDE), Ciudad de México. Retrieved from http://matchtec.mx/descargas/Plan\%20Ceibal.pdf

ECE (2014). Reporte Técnico. Evaluación Censal de Estudiantes. Retrieved from http://umc.minedu.gob.pe/resultadosece2016/

Escorcia-Oyola, L. I. \& De Triviño, C.A.J. (2015). Tendencias de uso de las TIC en el contexto escolar a partir de las experiencias de los docentes. Educación y Educadores, 18(1),137152. Retrieved from http://www.redalyc.org/articulo.oa?id=83439194008 [GS Search]

Hinojo, M. A. \& Fernández, A. (2012). El aprendizaje semipresencial o virtual: nueva metodología de aprendizaje en Educación Superior. Revista Latinoamericana de Ciencias Sociales, Niñez y Juventud, 10(1), 159-167. Retrieved from http://revistaumanizales.cinde.org.co/index.php/Revista-Latinoamericana/article/view/591 [GS Search]

Ibarra, M. J., Ataucusi, P. \& Ataucusi E. (2015). EducaApurimac una plataforma educativa con múltiples recursos digitales para enseñar en escuelas rurales sin acceso a internet. Proceedings de X Conferência Latino-Americana de Objetos e Tecnologias de Aprendizagem - LACLO-Brasil, 26-35. Retrieved from http://www.brie.org/pub/index.php/teste/article/view/5782 [GS Search] 
Ibarra, M.J., Soto, W., Ataucusi, P. \& Ataucusi, E. (2016, November). MathFraction: Educational serious game for students motivation for math learning. 2016 XI Latin American Conference on Learning Objects and Technology (LACLO), San Carlos-Costa Rica, 2016, 1-9. doi: 10.1109/LACLO.2016.7751777

Kulkarni, Y., Ballal, S. \& Gawade, J. (2012). Technology Transfer to Rural Population through Secondary Schools: The Vigyan Ashram Experience. In Global Humanitarian Technology Conference GHTC, IEEE, 411-416. doi: 10.1109/GHTC.2012.59

LLECE (2014), Reporte Técnico. Laboratorio Latinoamericano de Evaluación de la Calidad de la Educación 2014. Retrieved from http://umc.minedu.gob.pe/llece/

Marchesi, A., Blanco, R., Hernández, L., \& Educativas, M. (2014). Avances y desafíos de la educación inclusiva en Iberoamérica. Espa-a: Organización de Estados Iberoamericanos para la Educación, la Ciencia y la Cultura (OIE). Retrieved from https://oeidominicana.org.do/wp-content/uploads/2014/09/Metas_inclusiva.pdf

Martínez Castro, M.L. (2015). La práctica del docente universitario con herramientas TIC: un nuevo desafío. Revista Iberoamericana de Producción Académica y Gestión Educativa, 2015(Enero-Junio). $\quad$ Retrieved

from http://www.pag.org.mx/index.php/PAG/article/view/472

Marzal, M. Á., Colmenero-Ruiz, M. J., \& Morato Lara, J. L. (2003). Selección de recursos didácticos en red: accesibilidad y usabilidad como elementos de un sistema de evaluación para la Educación. $2^{\text {a }}$ Conferencia Iberoamericana en Sistemas, Cibernética e Informática. (CISCI 2003), 115-120. Retrieved from http://hdl.handle.net/10016/13859

OECD (2007). Giving knowledge for free: the emergence of open educational resources. Retrieved from www.oecd.org/edu/ceri/38654317.pdf

Paredes R. (2010). La pobreza y su dinámica en el sur del Perú. Informe final de estudio del Consorcio de Investigación Económica y Social y la UNA-Puno. Retrieved from http://repositorio.minedu.gob.pe/handle/123456789/1301

PISA (2017). Reporte Técnico. Programme for International Student Assessment, Retrieved from http://umc.minedu.gob.pe/wp-content/uploads/ 2017/04/Libro_PISA.pdf.

Rivero, I. L., Padrón, A. L. \& Izaguirre, E.Z. (2012). Didáctica del uso de las TIC y los medios de enseñanza tradicionales en las Instituciones de Educación Superior (IES) municipalizadas. New approaches in educational research, 1(1). doi: 10.7821/naer.1.1.33$\underline{40}$

Santos Carrazana, A. \& Carrazana Quintero, G. (2015) Herramienta de ayuda al aprendizaje de la Matemática. Memorias VE2015, Guadalajara México. Retrieved from http://reposital.cuaed.unam.mx:8080/jspui/handle/123456789/4581 [GS Search]

Santos-Hermosa, G., Ferran-Ferrer, N. \& Abadal, E. (2012). Recursos educativos abiertos: repositorios y uso. El profesional de la información, 21(2), 136-145. Retrieved from http://hdl.handle.net/10609/13601 [GS Search]

Sunkel, G. \& Trucco, D. (2012). Las tecnologías digitales frente a los desafíos de una educación inclusiva en América Latina: Algunos casos de buenas prácticas. Naciones Unidas CEPAL, Chile. Retrieved from http://repositorio.cepal.org/handle/11362/21658

Tedesco, J.C. (1999). Educación y sociedad del conocimiento y de la información. Documento presentado en Encuentro internacional de educación media Secretaría de Educación de 
Bogotá, agosto. $\quad$ Retrieved from http://revistas.pedagogica.edu.co/index.php/RCE/article/view/5876

UNESCO (2012). Declaración de París de 2012 sobre los Recursos Educacionales Abiertos (junio 2012). Congreso Mundial sobre los Recursos Educativos Abiertos (REA). Paris, Junio.

Zabala, S., Zabala, S. \& Reyes, J. (2013). Proyecto Canaima Educativo...Hacia la Apropiación Tecnológica, Venezuela. 